\title{
NOTAS EN TORNO A UNOS CHORI RIXAMARVM EN MARCIAL*
}

\author{
NOTES ON CHORI RIXAMARVM IN MARTIAL
}

\author{
JAVIER RAMIA \\ Universidad de Granada \\ jramia@ugr.es \\ ORCID: 0000-0002-5898-7122
}

DOI: $10.1387 /$ veleia.18083

\begin{abstract}
Resumen: Entre los nomina duriora que Marcial nos ha legado en el epigrama 4.55 se encuentra el hápax Rixamae (Mart. 4.55.16: Tutelamque chorosque Rixamarum), un término tradicionalmente entendido como un topónimo celtibérico. Sin embargo, Gimeno y Rothenhöfer (2012) han propuesto su reinterpretación no como un topónimo, sino como un conjunto de divinidades. En este artículo se presentan diferentes argumentos que podrían corroborar dicha propuesta.

Palabras clave: Rixamae, nomina duriora, Marcial, teónimo, topónimo.

Abstract: The hapax Rixamae (Mart. 4.55.16: Tutelamque chorosque Rixamarum), one of the nomina duriora that Martial bequeathed us in the epigram 4.55, has been traditionally understood as a Celtiberian place name. Nonetheless, Gimeno and Rothenhöfer (2012) put forward its reinterpretation not as a place name, but as a set of deities. This paper aims to point out some different arguments which may corroborate that proposal.

Keywords: Rixamae, nomina duriora, Martial, theonym, place name.
\end{abstract}

Recibido: 16-03-2016

Informado: 21-07-2016

Definitivo: 01-09-2016

\section{INTRODUCCIÓN}

En el año 2012 Bermejo publicó un epígrafe votivo consagrado a las Ríxamas ${ }^{1}$, verosímilmente unas divinidades de raigambre céltica que eran, al menos en apariencia, desconocidas hasta la fe-

* Una primera versión de este trabajo se presentó en el XIV Congreso de Estudios Clásicos celebrado en la Universitat de Barcelona entre los días 13 y 18 de julio de 2015. Me gustaría mostrar mi agradecimiento, de un lado, a la fundación Ana María Aldama Roy por la concesión una beca para la asistencia al referido congreso y, de otro, al Dr. Xaverio Ballester por la revisión de versiones anteriores del presente artículo, de cuyos eventuales errores o inexactitudes soy el único responsable.

1 A pesar de que con alguna frecuencia leemos en nuestra lengua Rixamas, la versificación corrobora su acentuación proparoxítona en latín (Rixămae) y, por supuesto, también en español (Ríxamas). 
cha. Reproducimos a continuación el texto que no presenta en principio dificultad alguna de lectura e interpretación:

\author{
IVNIA $\cdot$ AVITA \\ RIXAMIS \\ $A($ nimo $) \cdot L($ ibens $) \cdot D($ edit $)$
}

Aunque Bermejo no hace en ese artículo explícita mención a ello, el conjunto de divinidades que aparece en la inscripción aruccitana habría de remitir a los enigmáticos chori Rixamarum (Mart. 4.55.16: Tutelamque chorosque Rixamarum) que tenemos documentados únicamente en un verso de Marcial. La referencia a dichos coros se encuentra en el epigrama 4.55, un poema que constituye, junto con el 1.49, una fuente de extraordinario valor para conocer algunos vocablos relativos a su Celtiberia natal que tradicionalmente han sido interpretados en su totalidad como topónimos².

Pues bien, la vinculación entre los chori Rixamarum y las divinidades a las que se dedica la inscripción no pasa desapercibida a Gimeno y Rothenhöfer (2012), quienes no sólo ponen de manifiesto la mención de un hápax de la patria de Marcial en una inscripción latina ${ }^{3}$, sino que además hacen una propuesta ciertamente sugestiva: a la luz del testimonio epigráfico, la voz Rixamae atestiguada en Marcial no haría referencia, como tradicionalmente se ha defendido, a un topónimo celtibérico, sino a un conjunto de divinidades.

La inscripción es, en este sentido, en buena medida elocuente. En todo caso, permítasenos soslayar el análisis en profundidad del epígrafe, ya realizado desde diferentes perspectivas por Bermejo (2012) y especialmente por Gimeno y Rothenhöfer (2012), a cuyos exámenes difícilmente podríamos aportar algún dato significativo. Por nuestra parte, nos proponemos exponer algunos argumentos que, aunque en modo alguno tan decisivos como la propia inscripción, podrían sustentar todavía más la interpretación en clave teonímica del término Rixamae en Marcial.

\title{
2. LA VISIÓN TRADICIONAL
}

Como apuntábamos, el vocablo Rixamae ha sido interpretado con práctica unanimidad como un topónimo. Así lo hacen, por ejemplo, Holder (1904, s.u. Rīx-ămae), Thiele (1911, 265), Dolç $(1953,216 ; 1957,78)$ o Tovar $(1989,418)$ y, de igual manera, así aparece en la entrada correspondiente de la hoja K-30 de las Tabulae Imperii Romani (Cepas 1993, s. u. Rixamae) y en el comentario de Moreno $(2006,395)$ al libro IV de los epigramas de Marcial. En esta senda, Dolç $(1953,218)$ defendía una equivalencia entre Rixamae y Sisamón, al mismo tiempo que lamentaba

2 A lo largo de la obra epigramática de Marcial encontramos, además de Rixamae (4.55.16), voces como las que siguen: Bilbilis $(1.49 .3$, 1.61.12, 4.55.11, 10.103.1, 10.104.6, 12.18.9), Boterdus (1.49.7, 12.18.11), Burado (4.55.23), Caius (1.49.5, 4.55.2), Carduae (4.55.17), Congedus (1.49.9), Dercenna (1.49.17), Nutha (1.49.18), Peteris (4.55.18), Platea (4.55.13, 12.18.11), Rigae (4.55.19), Salo (1.49.12, $4.55 .15,10.13 .1,10.96 .3,10.103 .2,10.104 .6,12.2 .3$, 12.21.1, 14.33.2), Tagus $(1.49 .15,4.55 .2,6.86 .5$,
7.88.7, 8.78.6, 10.17.4, 10.65.4, 10.78.12, 10.96.3, 12.2.3), Tuetonissa (4.55.22), Turasia (4.55.21), Turgontum (4.55.21), Tutela (4.55.16), Vadauero (1.49.6), Vatiuesca (4.55.26) y Voberca (1.49.14).

${ }^{3}$ Este hecho constituiría, por sí solo, un verdadero hito, puesto que solamente una inscripción publicada por Mayer (2000-2001) contiene otro de los hápax celtibéricos de nuestro epigramista: GENIO - CAMPI . KARDVAR(um) | HVIVS LOCI - C(aius) - IVLIVS | C(aii) . IVLI E EVODI ALVMNVS $\mid$ HERMETIO · PO(suit). 
que los comentaristas apenas hubiesen tratado de localizar esta — citamos literalmente- «ciudad amante de la diversión».

Ha habido, no obstante, alguna propuesta divergente. Como apuntan Gimeno y Rothenhöfer (2012, 437), constituye una excepción Sullivan $(1991,177)$, quien en su célebre monografía Martial, the Unexpected Classic entiende Rixamae no como nombre de lugar, sino como el etnónimo de una tribu cercana a Sisamón. Asimismo el propio Sullivan $(1991,177)$ aporta una explicación curiosa a propósito de los chori: «choros Rixamarum may refer to the rings and natural amphitheatres in which wild-beast fighting took place then as now». Aunque no somos del parecer de Sullivan, es en buena medida sugestivo que sea precisamente este término el que, a su juicio, debía merecer reinterpretación.

\section{Algunos argumentos para una nUeva interpretación}

Pues bien, en primer lugar deberíamos preguntarnos por qué se ha entendido de manera generalizada Rixamae como un topónimo. Una de las posibles causas radicaría en que podría esperarse que Marcial, siguiendo con el catálogo de lugares del epigrama 1.49, hubiese versificado en el 4.55 únicamente topónimos. Esta visión podría sustentarse además en el hecho de que en este epigrama los referentes celtibéricos, que con la excepción de Tagus (4.55.2) y Caius (4.55.2) se introducen bajo el rótulo nos Celtis genitos et ex Hiberis / nostrae nomina duriora terrae / grato non pudeat referre uersu (4.55.8-10), se oponen principalmente a célebres ciudades helénicas como Tebas (4.55.5), Micenas (4.55.5) o Rodas (4.55.6).

Con todo, no sería inverosímil algún paréntesis no toponímico dentro de dicho listado de lugares. De hecho, en el epigrama 4.55 aparecen también, después de la expresión nostrae nomina duriora terrae (4.55.9), algunos nombres que no constituirían topónimos (4.55.12: quae uincit Chalybasque Noricosque). De igual manera, en el catálogo de lugares celtibéricos del epigrama 1.49 - poema del que, en última instancia, dependería el presente y con el que comparte un gran número de similitudes (Citroni 1975, 155-157) — leemos en versos muy próximos dos teónimos grecorromanos: Pomona (1.49.8: Pomona quod felix amat) y Nymphae (1.49.10: mollesque Nympharum lacus). Este último detalle es, a nuestro juicio, particularmente relevante, puesto que no nos encontramos sólo ante un paréntesis no toponímico dentro del catálogo de emplazamientos celtibéricos del poema 1.49, sino ante la mención expresa a divinidades. En consecuencia, atendiendo a los numerosos paralelos entre dichos epigramas, no podría descartarse la posibilidad de que hallásemos un paréntesis análogo.

Otro motivo que probablemente habría llevado a interpretar el término Rixamae como nombre de lugar lo constituiría su examen eminentemente lingüístico y la existencia de paralelos toponímicos. En efecto, se ha vislumbrado en esta voz una forma de superlativo (Holder 1904, s. u. Rixx-ămae; Dolç 1953, 216) que tendríamos también con cierta frecuencia en algunos topónimos hispanocélticos (Ballester 2004, 49) como Vxama (Plin. nat. 3.27), Segisama (Flor. epit. 2.33.48) y otros. Ciertamente se trata de un argumento muy poderoso y que no debería obviarse. Sin embargo, esta característica por sí sola no podría probar con absoluta certeza que estemos ante un topónimo, sobre todo si, como es el caso, también disponemos de alguna divinidad femenina con dicha característica morfológica en ámbito céltico como, verbigracia, la diosa gala Belisama (Villar y Prósper 2005, 273), atestiguada en epigrafía latina: MINERVAE $\mid$ BELISAMAE $\mid$ SACRVM $\mid$ Q(uintus) VALERIVS $\mid$ MONTAN(us) | (e)X V(oto) (CIL XIII 8). Asimismo, como recuerda Untermann (2001, 202), el sufijo -isamo «es frecuente en la antroponimia hispano-céltica». De este modo, constataríamos 
que, aunque en buena medida frecuente en topónimos, el sufijo de superlativo podría aplicarse también a otra suerte de voces como, por ejemplo, teónimos.

En todo caso, en lo referente a la morfología, el rasgo quizá más llamativo de la voz Rixamae no sea el sufijo de superlativo céltico, sino más bien su forma plural, especialmente si se compara con los lugares del epigrama 1.49 y con el resto de nomina duriora del 4.55. En efecto, si analizamos los veintiún términos tradicionalmente interpretados (Dolç 1957, 72 y otros) como topónimos celtibéricos que hallamos en los epigramas de Marcial, solamente tres nomina duriora parecen tener la forma de femeninos plurales y dos de ellos aparecen en versos consecutivos, en idéntica posición y con análogos casos y funciones:

\section{Tutelamque chorosque Rixamarum et conuiuia festa Carduarum (4.55.16-17)}

Estas aparentes formas plurales ya llamaron la atención de estudiosos como Dolç $(1953,217)$, a quien le parecieron — citamos de nuevo literalmente_ «raras». Un examen muy superficial de los endecasílabos falecios de Marcial relacionaría estas formas en -ae con voces cuales Thebae (4.55.5) y Mycenae (4.55.5), dos topónimos helénicos que aparecen en el verso quinto de este mismo epigrama. Ahora bien, esta explicación u otras que vinculan estas formas con el uso romano (Thiele 1911, 265) no serían suficientes, entre otros motivos, en la medida en que sólo se aplicaría la referida analogía a un número muy limitado de términos. Asimismo, aunque sí encontramos algunos topónimos ${ }^{4}$ o etnónimos ${ }^{5}$ acabados en -ae en nuestra península, la presencia de nombres con esta característica en el presente epigrama no deja de ser ciertamente llamativa.

Así pues, la explicación de la forma plural Rixamae no estaría, tal como apuntábamos más arriba, en el componente helénico del epigrama ni en la adaptación de tales vocablos al uso romano. Tampoco, al menos según nuestro parecer, sería suficiente la existencia de algunos topónimos con esa terminación para determinar que Rixamae fuera un nombre de lugar, del mismo modo que la existencia de etnónimos como Zoelae y Arrotrebae no prueba que estemos ante un etnónimo. Parece, en efecto, más sencillo pensar que esta forma anómala —o, mejor dicho, una de las formas anómalas del epigrama 4.55- hace referencia no a un topónimo, sino más bien a un colectivo femenino, esto es, a un conjunto de Ríxamas.

Precisamente esa es la conclusión a la que nos conduce un examen cuidadoso del texto latino. En efecto, ante un sintagma como chorosque Rixamarum quizá lo más natural —al menos si no tuviésemos el resto de condicionantes - sería la interpretación de la segunda voz como un colectivo femenino que, por su parte, nos informaría acerca de los formantes de dichos coros y no de su procedencia, sobre todo teniendo en cuenta los paralelos que hallamos en el propio Marcial y en otros autores de la romanidad.

En efecto, en otros versos de Marcial encontramos el vocablo chorus - término, como pertinentemente recuerdan Gimeno y Rothenhöfer $(2012$, 437), para mayor singularidad empleado frecuentemente en contextos religiosos - con un sentido en buena medida semejante. El bilbilitano

4 Entre los topónimos, dejando de lado nombres de lugar que disponen de formas latinas, podemos mencionar, entre otros y a modo de ejemplo, los citados por Guerra (2002, 149): Bortinae (IA 451.4; pero

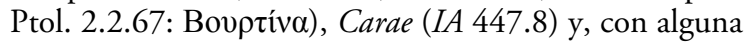

probabilidad, Tude (Plin. nat. 4.112; cf. Ptol. 2.6.45:

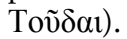

5 Entre los étnicos podemos añadir denominaciones como Arrotrebae (Plin. nat. 4.111.7) y Zoelae (Plin. nat. 3.28.1). 
versifica dicha voz en nueve ocasiones (sp. 26.1: lusit Nereidum docilis chorus aequore toto; 1.76.3: Pierios differ cantusque chorosque sororum; 4.44.4: hoc nuper Satyri monte dedere choros; 4.55.16: Tutelamque chorosque Rixamarum; 5.51.2: notariorum quem premit chorus leuis; 7.50.4: et Ganymedeo luceat unda choro; 7.69.8: quamuis Pierio sit bene nota choro; 10.62.3: et delicatae diligat chorus mensae; 12.2.8: reddita Pierio sunt ubi tecta choro) y en dos de ellas - si obviamos el verso que nos atañe- a chorus le sigue un genitivo plural femenino que indica sus formantes:

Pierios differ cantusque chorosque sororum (1.76.3)

lusit Nereidum docilis chorus aequore toto (sp. 26.1)

Prestemos atención a estos dos versos. En el primero encontramos un coro del que forman parte unas hermanas (chorosque sororum), a saber, las musas y del último las formantes son las nereidas (chorus Nereidum). Constatamos, pues, la presencia en Marcial del sustantivo chorus seguido de un genitivo plural (sororum, Nereidum) que identifica los miembros de dichas danzas en contexto mitológico. De hecho, es este un tratamiento muy frecuente en la literatura romana. Si extendemos nuestro examen más allá de Marcial, los resultados obtenidos no son en modo alguno distintos. Sirvan como ejemplo estos diez versos:

Musarum chorus et citharae pulsator Apollo (Flac. 5.693)
Nympharumque leues cum Satyris chori (Hor. carm. 1.1.31)
exercet Auster, Pleiadum choro (Hor. carm. 4.14.20)
et nemorum siluae Naiadumque chori (Ou. fast. 1. 512)
in studiis uitam Pieridumque choro (Ou. trist. 5.3.10)
Herculis Antaeique, Hesperidumque choros (Prop. 3.22.10)
Musarumque choris implicuisse manus (Prop. 3.5.20)
Nereidumque choris Cadmeia cingitur Ino (Sen. Oed. 446)
Nereidum chorus et sueto certamine nandi (Sil. 3.413)
Nereidum Phorcique chorus Panopeaque uirgo (Verg. Aen. 5.240)

Así pues, observamos que en los versos que hemos recogido a modo de ejemplo el genitivo plural no indica en ningún caso el origen, sino las — nótese el género del artículo - formantes de los coros, unas formantes que en todos los casos referidos son divinidades. De este modo, consideramos que una interpretación más certera del sintagma chorosque Rixamarum constituiría un argumento de mucho peso para la interpretación de Rixamae en Marcial no como un topónimo, sino más bien como un conjunto de divinidades femeninas.

Volvamos, para concluir el apartado, sobre el análisis lingüístico y la búsqueda de paralelos, labor de la que ya se ocupan Gimeno y Rothenhöfer $(2012,437)$ siguiendo esencialmente a Holder $\left(1904\right.$, s. u. Rixx-ămae). La voz Rixamae está formada por la raíz céltica ${ }^{*}$ rig- seguida de un sufijo de superlativo, formación para la que existe un "curioso paralelo» (Dolç 1953, 217): Rigisamus. Se trata de un epíteto de Marte - frecuentemente aducido, por cierto, cuando se examina nuestro vocablo- documentado en el inicio de dos inscripciones procedentes de Britania y Aquitania (CIL VII 61: DEO MARTI | RIGISAMO; CIL XIII 1190: MARTI | RIGISAMO). En consecuencia, disponemos también de un paralelo que, aunque señalado en alguna ocasión como un apunte "curioso», constituiría un argumento digno de ser tomado en consideración para la reinterpretación de Rixamae en clave teonímica.

Es más, el significado de nuestro término, extrapolable del que Holder (1904 s. u. Rĩğ̈-sămus) aplica al epíteto de Marte, «most royal or kingly», resultaba difícilmente explicable para Dolç 
(1953, 217-218), quien llegaba a preguntarse incluso si Rixamae "podría tratarse de una ciudad ennoblecida por los celtas con dicho apelativo divino». Sin embargo, tomando en consideración todos los datos de que disponemos, más bien podemos afirmar que el «apelativo divino» obedece al hecho de que las Rixamae constituyen en realidad un conjunto de divinidades femeninas, cuya vinculación con los coros tal vez podría relacionarse con un pasaje de Estrabón ${ }^{6}$ (3.4.16), en el

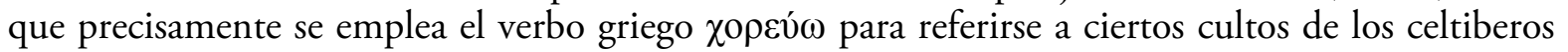
(Canto 1997, 35).

\section{Conclusiones y perspectivas}

En la presente contribución hemos tenido en cuenta argumentos de distinta naturaleza con los que hemos aspirado a corroborar en la medida de lo posible la propuesta de Gimeno y Rothenhöfer (2012). Con todo, debe tenerse presente que el argumento principal en que se sustentaba su trabajo, esto es, la inscripción aruccitana consagrada a las Ríxamas, era ciertamente decisivo.

Por lo demás, sería a nuestro juicio interesante que nos replanteásemos la naturaleza toponímica de otros nomina duriora de Marcial. En efecto, atendiendo esencialmente a argumentos de cariz morfológico y de cercanía en el epigrama 4.55, podrían ser merecedores de reinterpretación femeninos plurales como Carduae (4.55.17: et conuiuia festa Carduarum), cuyo paralelismo con las Ríxamas ya hemos subrayado supra y Rigae (4.55.19: atque antiqua patrum theatra Rigas), un término que, además, compartiría raíz con la voz Rixamae. Del mismo modo, podría ser también susceptible de examen particular Tutela (4.55.16: Tutelamque chorosque Rixamarum), vocablo que se encuentra en el mismo verso que las Ríxamas y para el que se han planteado tres posibilidades de interpretación: un nombre común, un topónimo o un teónimo.

Se trata, en suma, de una hipótesis de trabajo a nuestro juicio muy atractiva sobre la que será necesario seguir avanzando en próximos trabajos.

\section{BiBLIOGRAFÍA}

Ballester, X., 2004, "Páramo o del Problema de la */p/ en Celtoide», Studi Celtici 3, 45-56.

Bermejo, J., 2012, "Nuevas inscripciones aruccitanas: los Iulii, Iunii y otras gentes del territorio aruccitano», en: M. de Deus (ed.), Actas del V Encontro de Arqueologia do Sudoeste Peninsular, Almodôvar: Município de Almodôvar, 433-439.

Canto, A.M., 1997, «La tierra del toro. Ensayo de identificación de ciudades vasconas», AEspA 70, 31-70.

Cepas Palanca, A., 1993, «Rixamae», en: Tabula Imperii Romani. Hoja K-30: Madrid, Caesaraugusta, Clunia, Madrid: CSIC, 192.

Citroni, M., 1975, M. Valerii Martialis epigrammaton Liber Primus. Introduzione, testo, apparato critico e commento, Firenze: La nuova Italia.

Dolç, M., 1953, Hispania y Marcial. Contribución al conocimiento de la España antigua, Barcelona: CSIC.

—, 1957, «La investigación sobre la toponimia hispana de Marcial», EClás 4, 68-79.

6 "Algunos dicen que los galaicos no tienen dioses, y
que los celtiberos y sus vecinos del norte hacen sacrifi-
cios a un dios innominado, de noche en los plenilunios, ante las puertas, y que con toda la familia danzan y velan hasta el amanecer» (traducción de Meana y Piñero 1992, 108). 
GIL, J., 2004, «Marcial en Hispania», Humanitas 56, 225-326.

Gimeno Pascual, H., Rothenhöfer, P., 2012, «Eine neue Weihung an die Rixamae in der Baeturia Celticorum und Martial IV 55», Veleia 29, 435-439.

Guerra, A., 2002, "Omnibus Numinibus et Lapitearum: algumas reflexões sobre a nomenclatura teonímica do Ocidente peninsular», RPA 5 (1), 147-159.

Holder, A., 1904, Alt-celtischer Sprachschatz. Zweiter Band (I-T), Leipzig: Teubner.

Howell, P., 1980, A Commentary on Book One of the Epigrams of Martial, London: The Athlone Press.

Mayer, M., 2000-2001, "Kardvae, un testimonio de Marcial comprobado por la epigrafía», Zephyrus 5354, 529-534.

Meana, M.J., Piñero, F., 1992, Estrabón. Geografía (libros III-IV), [Biblioteca Clásica Gredos 169], Madrid: Gredos.

Moreno Soldevila, R., 2006, Martial, Book IV. A commentary, [Mnemosyne Supplement 278], Leiden: Brill.

Sullivan, J.P. , 1991, Martial: The Unexpected Classic: A Literary and Historical Study, Cambridge: Cambridge University Press.

Thiele, G., 1911, «Spanische Ortsnamen bei Martial», Glotta 3, 257-266.

Tovar, A., 1989, Iberische Landeskunde III: Tarraconensis, Baden-Baden: Valentin Koerner.

Untermann, J., 2001, «La toponimia antigua como fuente de las lenguas hispano-celtas», PalHisp 1, 187218.

Villar, F., Prósper, B.M., 2005, Vascos, celtas e indoeuropeos. Genes y lenguas, Salamanca: Ediciones Universidad de Salamanca. 